\section{PCR, Southern Blotting and Oocyte Expression to Assess the Efficacy of Subtractive Hybridization}

\section{BioTechniques 21:994-996 (December 1996)}

Subtractive hybridization is a technique designed to isolate genes and transcripts abundant in or unique to a particular condition. The method has been utilized for constructing a subtracted cDNA library, differential cloning and identifying differentially expressed genes in specific cell lines $(3-6,8,9)$. The analysis of the transcripts isolated by using this technology can be most difficult, in that even a subtracted library invariably contains an abundance of common transcripts. We therefore used the polymerase chain reaction (PCR), Southern blot analysis and the Xenopus laevis oocyte expression system to ascertain the efficacy of the subtraction technique.

Our goal was to utilize and expand the method as an alternative to traditional cloning techniques to facilitate the isolation of a growth-regulated renal sodium-dependent phosphate (NaPi) co-transporter, which we speculated was more abundant in the renal cortex of growing (3-week-old) than in adult (>12-week-old) animals. Due to the differences that exist in the renal handling of Pi between dietary Pi depletion and growth, we postulate that a transporter encoded by a growth-regulated mRNA different from $\mathrm{NaPi}-2$, the transporter modulated during dietary $\mathrm{Pi}$ deprivation, accounts for the high rates of renal Pi transport observed during growth (7). Northern blot analysis performed in our laboratory had already confirmed that the expression of NaPi2 was greater in the renal cortex of adult than in growing animals (1). Our aim was therefore to eliminate the common NaPi-2 transcripts and ascertain the presence of a novel and up-regulated $\mathrm{NaPi}$ transcript in the renal cortex of growing rats.

The protocol for subtractive hybridization used in our experiments was adapted from that of López-Fernández and Del Mozo (4). Hybridization was sought between cDNA derived from renal cortex of $>12$-week-old rats (the "driver" condition) and poly(A) RNA obtained from the renal cortex of 3week-old rats (the "tester" condition). Briefly, $10 \mu \mathrm{g}$ poly(A) RNA from the renal cortex of $>12$-week-old rats were isolated on magnetic particles and used for first-strand cDNA synthesis. The cDNA generated binds to streptavidincoated magnetic particles (Promega, Madison, WI, USA). Poly(A) RNA (10 $\mu \mathrm{g}$ ) isolated from the renal cortex of 3week-old rats was added to allow annealing of the mRNA to the cDNA for $20 \mathrm{~min}$ at $55^{\circ} \mathrm{C}$. The subtracted poly(A) RNA after each passage was collected, and some aliquots were used for reverse transcription (RT)-PCR and Southern blotting, while aliquots after

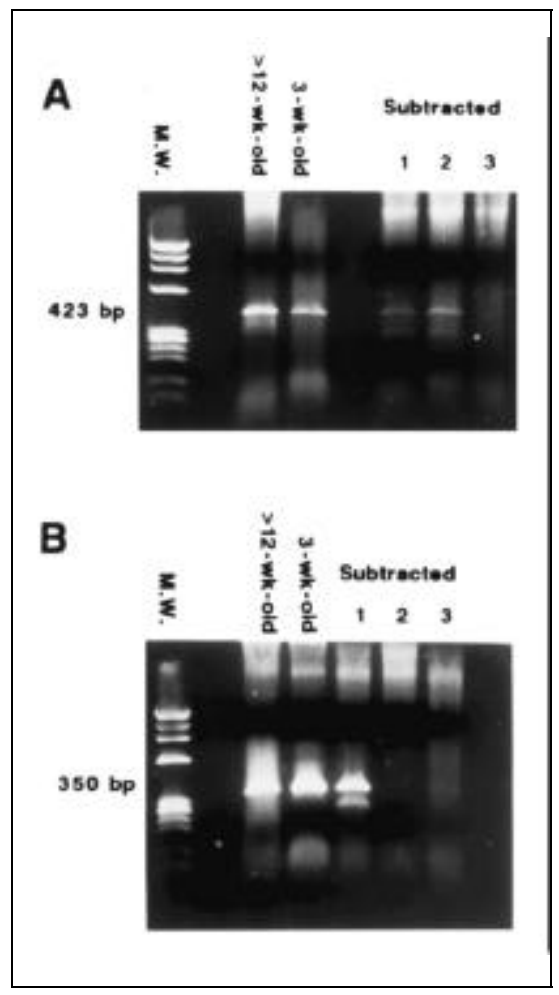

Figure 1. RT-PCR amplification products before and after subtractive hybridization. (A) RT-PCR of pre-subtracted renal cortical poly(A) RNA (3- and 12-week-old) and that of poly(A) RNA obtained from the renal cortex of 3 -week-old rats exposed to hybridization $(1,2$ and 3 cycles) with cDNA synthesized from $>12-$ week-old rats, using primers specific for $\mathrm{NaPi}-2$. (B) The same templates as in (A), assessing the presence of the control gene, $\beta$-actin. Note the elimination of $\mathrm{NaPi}-2$ and the control, $\beta$-actin, after three cycles of subtraction. the third passage were injected into oocytes.

RT-PCR (2) products for NaPi-2 (sense, position 1544-1565, CATGGCCAAGGCACTGGGCAAA) and (antisense, position 1944-1966, TAGAGCCGGGTGGCATTGTGGTG) (6) (423 bp) were abundant in the renal cortex of unsubtracted 3- and 12-week-old rats (internal control) (Figure 1A). RT-PCR for $\beta$-actin (control) of the same samples resulted in abundant product in the unsubtracted samples and steady removal after two subtracted cycles (Figure 1B). Because residual NaPi-2-specific RT-PCR product might have escaped detection because of lack of sensitivity of ethidium bromide staining, we blotted the gels (Figure 1A) onto nylon membranes and carried out Southern hybridization using a [ $\left.{ }^{32} \mathrm{P}\right] \mathrm{dCTP}-\mathrm{la}-$ beled full-length cDNA NaPi-2 probe. The results (Figure 2) confirm that poly(A) RNA, subjected to three cycles of subtractive hybridization, is completely depleted of $\mathrm{NaPi}-2$ transcripts.

With knowledge that NaPi-2 could be effectively removed by subtractive hybridization, we investigated whether a conserved region of another $\mathrm{NaPi}$ cotransporter was present within the renal cortex of 3-week-old animals. RT-PCR amplification products of unsubtracted 12- and 3-week-old renal cortical poly(A) RNA and 3-week-old poly(A) RNA exposed to subtractive hybridization with 12-week-old renal cortical cDNA are shown in Figure 2. The primers used in these experiments were those specific for $\mathrm{NaPi}-2$, and in addition, primers specific for a conserved region of all modulated (type II) $\mathrm{NaPi}$

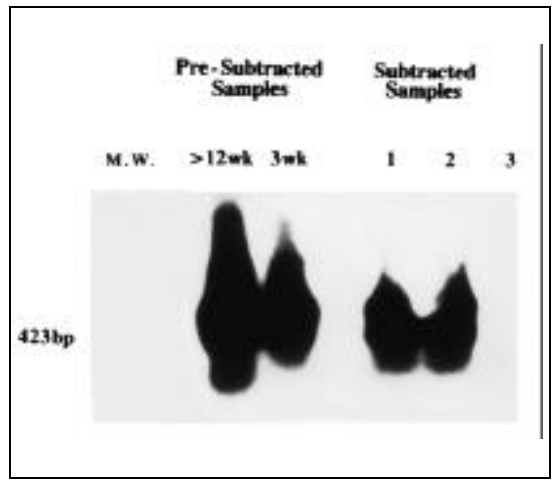

Figure 2. Southern blot. Blot from the ethidium bromide-stained gel from Figure 1A. Data are consistent with RT-PCR (Figure 1A). 


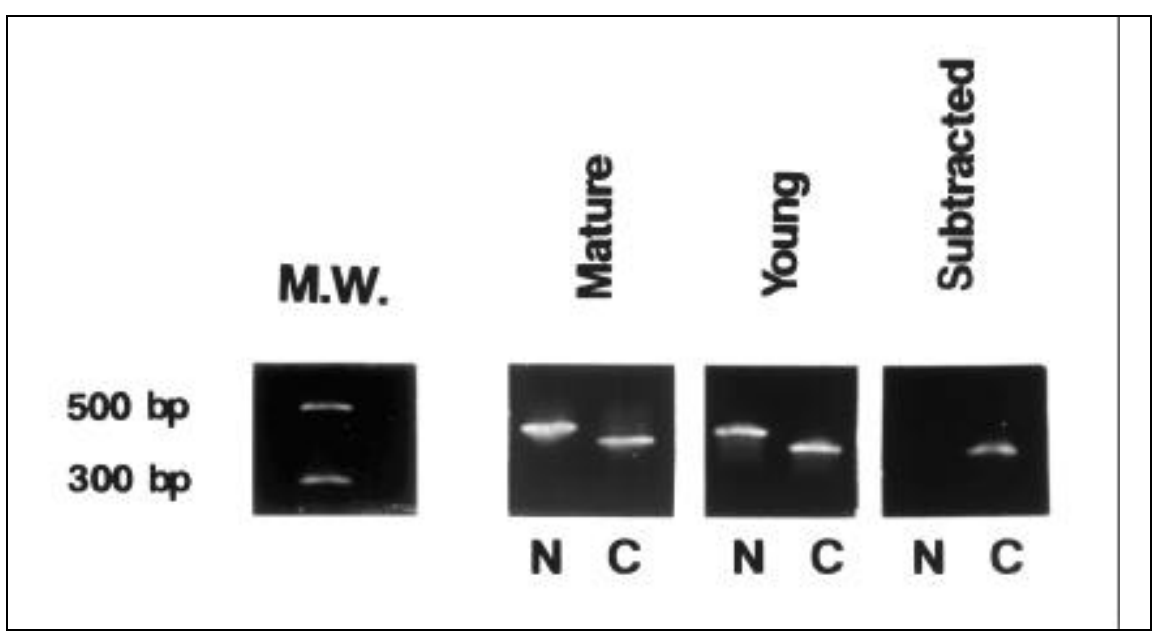

Figure 3. RT-PCR amplification products for NaPi-2 and the conserved region. Pre-subtracted $>12$ week-old and 3-week-old poly(A) RNA and 3-week-old poly(A) RNA exposed to subtractive hybridization with >12-week-old renal cortical cDNA were used as templates. Primers for $\mathrm{NaPi}-2(\mathrm{~N})$ and for a conserved region of Type II NaPi transporters (C). Note the absence of $\mathrm{NaPi}-2$ and the presence of the conserved region in the subtracted samples.

transporters (sense, positions 1278 1300, AGCATGACGTTTGTTGTCCAGAG) and (antisense, positions 1645-1657, CACAAACCGTAAAGATACCGTCC). The results revealed apparent removal of all NaPi-2 mRNA transcripts after subtraction while the expression of the conserved region was abundant in all samples (Figure 3). One obvious question remains: if $\mathrm{NaPi}-2$ and the putative transcript both contain this conserved region, why are both not removed by subtraction? First, note that the conserved region represents only a small segment of both $\mathrm{NaPi}-2$ and the putative transcript. Since subtractive hybridization depends upon specific annealing of large segments of transcripts, this important but, nevertheless, relatively small region of homology will not result in removal of all transcripts with this region in common. In addition, we considered it likely that the growth-regulated co-transporter is significantly more abundant in 3-weekold rat renal cortex. Hence, as demonstrated by Mészáros et al. (5), it is unlikely that it can be completely subtracted out of 3-week-old renal cortical poly(A) RNA.

By sequence analysis, the RT-PCR amplification product that escaped subtraction was found to be $98 \%$ homologous with the corresponding segment of NaPi-2 cDNA.

To ascertain the effect of subtractive hybridization on the capacity of the poly(A) RNA from the young animal to encode for $\mathrm{Na}^{+}-\mathrm{Pi}$ co-transport, we injected $50-\mu \mathrm{g}$ samples into oocytes and measured the rates of $\mathrm{Na}^{+}-\mathrm{Pi}$ uptake. Fifty nanoliters of diethylpyrocarbonate-treated water were injected as a control. No difference in $\mathrm{Na}^{+}$-dependent Pi uptake was observed $(P>0.3)$ between oocytes injected with poly(A) RNA harvested after the third subtractive passage ( $253 \pm 35 \mathrm{pmol} /$ oocyte $\cdot \mathrm{h})$ and those injected with poly(A) obtained before subtraction $(230 \pm 40$ $\mathrm{pmol} /$ oocyte $\mathrm{h}$ ). Both uptake rates were greater than that induced by poly(A) RNA of adult animals ( $163 \pm 34 \mathrm{pmol} /$ oocyte h, $P<0.05)$ and that observed in water-injected oocytes $(81 \pm 14 \mathrm{pmol} /$ oocyte $\cdot h, P<0.01)$. Therefore, poly(A) RNA of the young, depleted of NaPi-2 mRNA by subtractive hybridization, preserved its ability to encode for $\mathrm{Na}^{+}-$ Pi co-transport. The transcript detected in the subtracted sample by using primers designed to amplify a highly conserved region of the NaPi-2 mRNA, very likely represents part of the message for a growth-specific $\mathrm{Na}^{+}-\mathrm{Pi}$ cotransporter.

In conclusion, the PCR method in conjunction with Southern blot analysis and oocyte expression enhances one's ability to test the validity of subtractive hybridization. In addition, the method has permitted the isolation of a sub- tracted cDNA library, which can be screened by routine means.

\section{REFERENCES}

1.Barac-Nieto, M., B. Zavilowitz and A. Spitzer. 1994. A putative growth-modulated renal $\mathrm{Na}^{+}-\mathrm{Pi}$ cotransporter. J. Amer. Soc. Nephrol. 5:859.

2.Custer, M., M. Lötscher, J. Biber, H. Murer and B.Kaissling. 1994. Expression of Na-Pi transport in rat kidney: localization by RTPCR and immunohistochemistry. Am. J. Physiol. 35:F767-F774.

3.Hara, E., T. Kato, S. Nakada, S. Sekiya and K. Oda. 1991. Subtractive cDNA cloning using oligo(dT)30-latex and PCR: isolation of cDNA clones specific to undifferentiated human embryonal carcinoma cells. Nucleic Acids Res. 19:7097-7104.

4.López-Fernández, L.A. and J. Del Mozo. 1993. Construction ofsubtractive cDNA libraries from limited amounts of mRNA and multiple cycles of subtraction. BioTechniques 15:654-659.

5.Mészáros, M. and D.B. Norton. 1996. Subtractive hybridization strategy using paramagnetic olig(dT) beads and PCR. BioTechniques 20:413-419.

6.Raineri, I., C. Moroni and H.P. Senn. 1991. Improved efficiency for single stranded PCR by creating a reusable pool of first-strand cDNA coupled to a solid phase. Nucleic Acids Res. 19:4010.

7.Silverstein, D.M., M. Barac-Nieto and A. Spitzer. 1996. Mechanism of renal phosphate transport during growth. Kidney Int. 49:10231026.

8.Timblin, C., J. Battey and W.M. Kuehl. 1990. Application of PCR technology to subtractive cDNA cloning: identification of genes expressed specifically in murine plasmacytoma cells. Nucleic Acids Res. 18:1587-1595.

9.Weiland, I., G. Bolger, G. Asouline and M. Wigler. 1990. Method for difference cloning: gene amplification following subtractive hybridization. Proc. Natl. Acad. Sci. USA 87: 2720-2724.

Address correspondence to Douglas $M$. Silverstein, 1410 Pelham Parkway South, Rose F. Kennedy Center, Room 721, Bronx, NY 10461, USA.

Received 17 November 1995; accepted 3 May 1996.

Douglas M. Silverstein, Stefan Somlo, Beth Zavilowitz and Adrian Spitzer Albert Einstein College of Medicine Bronx, NY, USA 